\title{
Protective effect of hydrogen sulfide on renal injury in the experimental unilateral ureteral obstruction
}

\author{
Murat Dursun ${ }^{1}$, Alper Otunctemur ${ }^{2}$, Emin Ozbek ${ }^{3}$, Suleyman Sahin ${ }^{2}$, Huseyin Besiroglu ${ }^{2}$, Ozgur Doga \\ Ozsoy ${ }^{4}$, Mustafa Cekmen ${ }^{4}$, Adnan Somay ${ }^{5}$, Nurver Ozbay ${ }^{5}$ \\ 1 Department of Urology, Bahcelievler State Hospital, Istanbul, Turkey; ${ }^{2}$ Department of Urology, \\ Okmeydani Training and Research Hospital, Istanbul, Turkey; ${ }^{3}$ Department of Urology, Katip Celebi \\ University, Ataturk Training and Research Hospital, Izmir, Turkey; ${ }^{4}$ Department of Biochemistry, Kocaeli \\ University, Kocaeli, Turkey; ${ }^{5}$ Department of Pathology, Fatih Sultan Mehmet Training and Research \\ Hospital, Istanbul, Turkey
}

\section{ABSTRACT}

Introduction/Objective: Ureteral obstruction is a common pathology and causes kidney fibrosis and dysfunction at late period. In this present study, we investigated the antifibrotic and antiinflammatory effects of hydrogen sulfide on kidney damage after unilateral ureteral obstruction (UUO) in rats.

Materials and Methods: 24 rats were divided into four groups. Group 1 was control, group 2 was sham, group 3 included rats with UUO and group 4 rats with UUO which were given sodium hydrogen sulfide (NaHS)-exogenous donor of hydrogen sulfide (intraperitoneally $56 \mu \mathrm{moL} / \mathrm{kg} /$ day). After 14 days, rats were killed and their kidneys were taken and blood analysis was performed. Tubular necrosis, mononuclear cell infiltration and interstitial fibrosis were determined histopathologically in a part of the kidneys; nitric oxide (NO), malondialdehyde (MDA) and reduced glutathione (GSH) levels were determined in the other part of the kidneys. Urea-creatinine levels were investigated by blood analysis. Statistical analyses were made by the Chi-square test and one-way analysis of variance (ANOVA).

Results: There was no significantly difference for urea-creatinine levels among groups. Pathologically, there was serious tubular necrosis and fibrosis in group 3 and there was significantly decreasing of tubular necrosis and fibrosis in group $4(\mathrm{p}<0.005)$. Also, there was significantly increase of NO and MDA levels and decrease of GSH levels in group 3 compared to other groups $(\mathrm{p}<0.005)$.

Conclusions: hydrogen sulfide prevents kidney damage with antioxidant and antiinflammatory effect.

\section{ARTICLE INFO}

\section{Key words:}

Ureteral Obstruction; Hydrogen Sulfide; Nephrogenic Fibrosing Dermopathy; Oxidative Stress

Int Braz J Urol. 2015; 41: 1185-93

Submitted for publication:

February 02, 2014

Accepted after revision:

May 13, 2014

\section{INTRODUCTION}

Obstructive nephropathy is a common cause of renal insufficiency in children and adults. Decreases in renal blood flow and glomerular filtration occur after obstruction. Increased hydrostatic pressure causes damage to the tubule-inter- stitial compartment of the kidney (1). Apoptosis in tubular cells, capillary rarefaction, and interstitial cell inflammatory infiltration can be observed. The ensuing progressive fibrosis results in loss of parenchyma $(2,3)$. The obstruction can occur at any level of the urinary tract. The most common cause of obstruction in adults is urolithiasis, while 
obstructive nephropathy in children is mostly congenital (4).

Unilateral ureteral obstruction (UUO) is a well-established model known to imitate the process of obstructive nephropathy in a simple, accelerated and species-independent manner (5). In recent years, recovery of renal morphology following the relief of unilateral ureteral obstruction (UU0) has been examined in neonatal rats. Interestingly, it has been demonstrated that progressive tubule-interstitial and glomerular damage persisted in the obstructed and contralateral kidney and a decrease in glomerular filtration rate (GFR), and an increase in proteinuria occurred at the end of 1 year after relief of UUO $(5,6)$. Reactive oxygen species (ROS) are a recently recognized mechanism in the pathogenesis of UUO in experimental studies (7). Increased lipid peroxidation has been reported in renal cortexes of UUO animals. It has been shown that oxidative stress in UUO contributes to the development of tubulo-interstitial lesions and renal fibrosis. Various factors with complex cellular and molecular interactions have also been proposed as possible causes that lead to tubulo-interstitial lesions and renal fibrosis (8). Consequently, new therapy approaches are needed to prevent progression of renal injury along with surgical intervention. Therefore, concomitant treatment with an antifibrotic agent at the time of relief of UUO may prevent deterioration of renal function due to fibrosis. As previously reported, one of these agents may be hidrogen sulfide $\left(\mathrm{H}_{2} \mathrm{~S}\right)$. For decades, hydrogen sulfide $\left(\mathrm{H}_{2} \mathrm{~S}\right)$ has been known as a toxic gas, and, together with nitric oxide (NO) and carbon monoxide (CO), it is currently recognized as an endogenous gaseous physiological molecule (9). $\mathrm{H}_{2} \mathrm{~S}$ is synthesized from cysteine by two pyridoxal-5'-phosphate dependent enzymes, cystathionine $\beta$-synthase (CBS) and cystathionine $\gamma$-lyase (CSE), and a pyridoxal-5'-phosphateindependent enzyme, 3-mercaptpyruvate sulfurtransferase (3-MST), in most mammalian tissues, including the kidney $(10,11)$. Progression of fibrosis is associated with oxidative stress, inflammatory response, vascular tone, and intracellular signaling pathways. Recent studies in human and animal have demonstrated involvement of $\mathrm{H}_{2} \mathrm{~S}$ in those factors in various diseases, including ath- erosclerosis, ischemia and reperfusion (I/R) injury, hypertension, and end-stage renal disease (ESRD) $(10,11)$. In a previous study, $\mathrm{H}_{2} \mathrm{~S}$ supplementation was associated with the suppressions of oxidative stress, inflammation and nitrosative stress (12).

Because of these effects of $\mathrm{H}_{2} \mathrm{~S}$, in this study we investigated the role of $\mathrm{H}_{2} \mathrm{~S}$ in renal damage due to UUO. We used an exogenous donor of hidrogen sulfide-sodium hidrogen sulfide. We evaluated the antifibrotic, antinflammatory and antioxidative effects of $\mathrm{H}_{2} \mathrm{~S}$ in rat kidneys.

\section{MATERIALS AND METHODS}

\section{Drugs and Animals}

Male Wistar albino rats (200-250 g) were housed in clean plastic cages in a temperature and humidity-controlled facility with a constant $12 \mathrm{~h}$ light/dark cycle with free access to food and water. The use of animals and the experimental protocol were approved by the Institutional Animal Care and Use Committee and animals were treated in accordance with the Guide for the Care and Use of Laboratory Animals of Research Council. Like previous study, sodium hydrogen sulfide (NaHS)-exogenous donor of $\mathrm{H}_{2} \mathrm{~S}$ (Merck, Schuchardt, OHG, 85662 HOHENBRUNN, Germany), was administered intraperitoneally $56 \mu \mathrm{moL} / \mathrm{kg} /$ day for 14 days (13).

\section{Experimental design}

One week after acclimatization, UUO was induced. Briefly after induction of general anesthesia by intraperitoneal injection of thiopental $(100 \mathrm{mg} / \mathrm{kg})$, the abdominal cavity was exposed via midline incision and the left ureter was ligated at 2 points with 4-0 silk. The sham-operated rats had their ureters manipulated but not ligated. All rats were given amikacin sulfate $(6 \mathrm{mg} / \mathrm{kg}$, intramuscularly route) before operation (14).

After a quarantine period of 7 days, 24 rats were randomly divided into four groups, each consisting of six animals as follows: Rats in group 1 were control; Rats in group 2 were submitted to sham operation; Rats in group 3 underwent unilateral ureteral ligation and received no treatment; Rats in group 4 were subjected to unilateral ureteral ligation and received NaHS (intraperitone- 
ally $56 \mu \mathrm{moL} / \mathrm{kg} /$ day ) for 14 days. At this time, no animals showed symptoms of pyonephrosis and no one died because of pyonephrosis. So, we did not have to replace any animals. After 15 days, rats were killed and their kidneys were taken and blood analysis was performed. Tubular necrosis, mononuclear cell infiltration and interstitial fibrosis scoring were determined histopathologically in a part of kidneys; nitric oxide (NO), malondialdehyde (MDA) and reduced glutathione (GSH) levels were determined in the other part of the kidneys. Urea and creatinine levels were investigated by blood analysis.

\section{Biochemical Assays}

Twenty four hours after the administration of the last doses of NaHS, on 15th day, rats were anesthetized by intraperitoneal injection of ketamine and sacrificed. Renal cortical tissues were separated into two parts for biochemical analysis and light microscopic examination. Blood samples were also taken by cardiac puncture to assess the serum levels of urea and creatinine concentrations. In frozen tissues biochemically malondialdehyde (MDA), end product of lipid peroxidation, reduced glutathion (GSH), nonenzymatic antioxidant, and total nitrite, a stable product of nitric oxide (NO), were evaluated as a means of oxidative stress. Renal impairment was assessed by serum urea and creatinine levels, as well as by the kidney histology. Serum urea and creatinine levels were determined with an autoanalyzer (Syncron LX20, Ireland) by using commercial Becman Coulter diagnostic kits. Kidney tissue (300mg) was homogenized in icecold tamponade containing $150 \mathrm{mM}$ KCL for determination of MDA. MDA levels were assayed for products of lipid peroxidation. MDA referred to as thiobarbituric acid reactive substance, was measured with thiobarbituric acid at $532 \mathrm{~nm}$ using a spectrofluorometer, as described previously. GSH was determined by the spectrophotometric method, which was based on the use of Ellman's reagent. Total nitrite (NOx) was quantified by the Griess reaction after incubating the supernatant with Escherichia coli nitrate reductase to convert $\mathrm{NO}_{3}$ to $\mathrm{NO}_{2}$. Griess reagent ( $1 \mathrm{~mL} \mathrm{1 \%} \mathrm{sulfanilamide,}$ $0.1 \%$ naphtyl-ethylenediamine hydrochloride, and 2.5\% phosphoric acid; Sigma Chemical Co., St.
Louis, MO, USA) was then added to $1 \mathrm{~mL}$ of supernatant. The absorbance was read at $545 \mathrm{~nm}$ after a 30-min incubation. The absorbance was compared with the standard graph of $\mathrm{NaNO}_{2}$, obtained from the reduction of $\mathrm{NaNO}_{3}(1-100 \mathrm{mmoL} / \mathrm{L})$. The accuracy of the assay was checked in two ways; the inter- and intraassay coefficients of variation were 7.52 and $4.61 \%$, respectively. To check conversion of nitrate to nitrite (recovery rate), known amounts of nitrate were added to control plasma samples; these samples were deproteinized and reduced as above.

\section{Histopathological Examinations}

Histopathological evaluation of the kidney tissues was done. Paraffin embedded specimens were cut into $6 \mu \mathrm{m}$ thickness and stained with Hematoxylin-Eosin stain for light microscopic examination using a conventional protocol (Olympus, BH-2, Tokyo, Japan). A semi-quantitative evaluation of renal tissues was accomplished by scoring the degree of severity according to previously published criteria (15). All sections of kidney samples were examined for tubular necrosis. Briefly, a minimum of 50 proximal tubules associated with 50 glomeruli were examined for each slide and an average score was obtained. Severity of lesion was graded from 0 to 3 according to the percentage of tubular involvement. Slides were examined and assigned for severity of changes using scores on scale in which (0) denotes no change; grade (1) changes affecting $<25 \%$ tubular damage (mild); grade (2) changes affecting $25-50 \%$ of tubules (moderate); grade (3) changes affecting $>50 \%$ of tubules (severe). Histopathological evaluation was performed on left kidney tissues. Paraffin-embedded specimens were cut into $5 \mathrm{~mm}$ thick sections and stained with Hematoxylin \&t Eosin and Masson's trichrome for examination under the light microscope (BH-2; Olympus, Tokyo, Japan). To evaluate leukocyte infiltration, the widening of interstitial spaces with focal leukocyte infiltration was assessed in five randomly chosen sections prepared from each kidney sample. For each section, the average number of leukocytes per $0.28 \mathrm{~mm}^{2}$ was calculated from these leukocyte-infiltrated foci using a high-power microscopic field (X400). To estimate the grade of interstitial fibro- 
sis, the interstitial area that was stained green with Masson's trichrome was evaluated as a percentage of the total examined area in five randomly chosen sections prepared from each kidney sample using an image analyzer (Leica; Leica Micros Imaging Solutions, Cambridge, UK). For each section, interstitial space widening with focal leukocyte infiltration and interstitial fibrosis was assessed in high-power fields (X400) to quantify the results. The Banff classification of kidney pathology was used for scoring the degree of mononuclear cell infiltration and interstitial fibrosis. The score was graded from 0 to 3 , depending on the severity of histological characteristics (16).

\section{Statistical analysis}

Results of all groups are shown as mean values \pm standard deviation (SD). Statistical analyses of the histopathologic evaluation of the groups were carried out by the Chi-square test and biochemical data were analyzed by the one-way analysis of variance (ANOVA). The significance between two groups was determined by the
Dunnett's multiple comparison test, and $\mathrm{P}<0.05$ was accepted as statistically significant value.

\section{RESULTS}

\section{Biochemical Variables in Plasma and Tissue}

There was no significantly difference for urea-creatinine levels between groups (Table-1). Tissue MDA levels significantly increased in group 3 compared with groups 1,2 , and $4(\mathrm{p}<0.05)$. Rats with NaHS administration (group 4) showed reduced levels of lipid peroxidation as measured by MDA levels (Table-2). UUO also induced a significant increase in the tissue NO levels that have been prevented by NaHS (Table-2). The unilateral ureteral ligation was accompanied by a marked reduction in GSH level in the kidney tissues of rats $(p<0.05)$, and treatment with NaHS partially elevated the GSH levels (Table-2).

\section{Histopathologic Examinations Results}

Histopathologic examination of kidney showed no pathologic findings in control group (Figure-1a). In rats with UUO, there were mild and

Table 1 - Effects of UUO alone and its combination with NaHS on plasma urea, creatinine levels in rats.

\begin{tabular}{lcccc}
\hline Parameters & $\begin{array}{c}\text { Control } \\
\text { (Group 1) }\end{array}$ & $\begin{array}{c}\text { Sham } \\
\text { (Grup 2) }\end{array}$ & $\begin{array}{c}\text { UO } \\
\text { (Grup 3) }\end{array}$ & $\begin{array}{c}\text { U0+NaHS } \\
\text { (Grup 4) }\end{array}$ \\
\hline Urea $(\mathrm{mg} / \mathrm{dL})$ & $29 \pm 7.4$ & $28.7 \pm 8.1$ & $30.2 \pm 9.5$ & $29.9 \pm 10.3$ \\
Creatinine $(\mathrm{mg} / \mathrm{dL})$ & $0.38 \pm 0.3$ & $0.36 \pm 0.1$ & $0.42 \pm 0.2$ & $0.40 \pm 0.1$ \\
\hline
\end{tabular}

Values are expressed as mean $\pm \mathrm{SD}$ for six rats in each group.

Table 2 - Effect of $\mathrm{H}_{2} \mathrm{~S}$ on the Levels of Malondialdehyde, Glutathione and Nitric Oxide in Each Rat Group.

\begin{tabular}{lcccc}
\hline Parameters & $\begin{array}{c}\text { Control } \\
\text { (Grup 1) }\end{array}$ & $\begin{array}{c}\text { Sham } \\
\text { (Grup 2) }\end{array}$ & $\begin{array}{c}\text { UO } \\
\text { (Grup 3) }\end{array}$ & $\begin{array}{c}\text { U0+NaHS } \\
\text { (Grup 4) }\end{array}$ \\
\hline NO (nmoL/g wet tissue) & $24.83 \pm 3.95$ & $24.63 \pm 3.28$ & $38.90 \pm 6.82^{\mathrm{a}}$ & $27.03 \pm 3.61^{\mathrm{b}}$ \\
MDA (nmoL/g wet tissue) & $2.75 \pm 0.19$ & $2.84 \pm 0.29$ & $4.17 \pm 0.79^{\mathrm{c}}$ & $3.07 \pm 0.45^{\mathrm{d}}$ \\
GSH (mg/g wet tissue) & $2.25 \pm 0.10$ & $2.25 \pm 0.04$ & $1.20 \pm 0.73^{\mathrm{e}}$ & $2.06 \pm 0.15^{\dagger}$ \\
\hline
\end{tabular}

Values are expressed as mean $\pm S D$ for six rats in each group

a,ce, significantly difference from control group $(p=0.02, p=0.005, p<0.001)$.

b,dif significantly difference from U0 group ( $p=0.006, p=0.015, p<0.001)$.

$\mathbf{N O}=$ nitric oxide, $\mathbf{M D A}=$ malondialdehyde, $\mathbf{G S H}=$ reduced glutathione 
severe tubular necrosis in the proximal tubules compared with control and sham groups (Figures $1 \mathrm{~b}$ and $1 \mathrm{c})$. In rats treated with UUO+NaHS, despite the presence of mild tubular degeneration and tubular necrosis, the findings were less severe, and glomeruli maintained a better morphology when compared with UUO group (Figure-1d). Histopathologic examination was normal in rats with only sham operation (group 2) and in rats with no operation (group 1). Severe leukocyte infiltration was observed in the periglomerular and peritubular interstitium of the kidneys of the rats in group 3 with UUO (Figures 2a and 2b). Quantitative analysis of the focal leukocyte infiltration area in the interstitium showed that leukocyte infiltration was significantly reduced in rats that received UUO+NaHS (group 4) (Figure-2c). UUO caused a significant interstitial fibrosis in rats that received no treatment (group 3), and the percentage area of interstitial fibrosis in the kidney of rats with UUO that received no treatment was significantly greater than that of rats with UUO that received NaHS (group 4) (Figures 3a, 3b and 3c). These changes are summarized in Table-3.

\section{DISCUSSION}

Obstructive uropathy, caused by prevention of urine flow, results in permanent renal damage and loss of renal function. The obstruction can occur at any level of the urinary tract. The most common cause of obstruction in adults is urolithiasis, while obstructive nephropathy in children is mostly congenital (4). Acute obstruction of the ureter rapidly triggers a cascade of events in the kidneys. First, renal blood flow and

Figure 1 - A) normal tubulus and glomerules in kidney kortex H\&Ex200 (control group). B) normal tubulus and glomerules in kidney kortex H\&Ex200 (sham group). C) severe tubules total necrosis, tutbular degeneration and epithelial vacuolization in proximal tubules H\&Ex200 (UUO group). D) mild epithelial vacuolization in the proximal tubules and normal glomerules H\&Ex2OO (UUO+NaHS treated group).
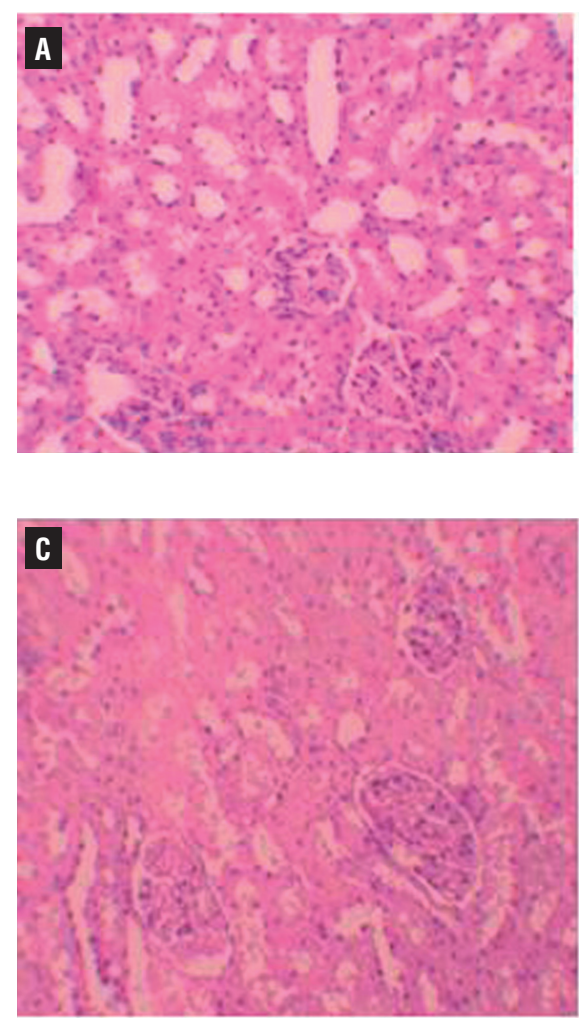
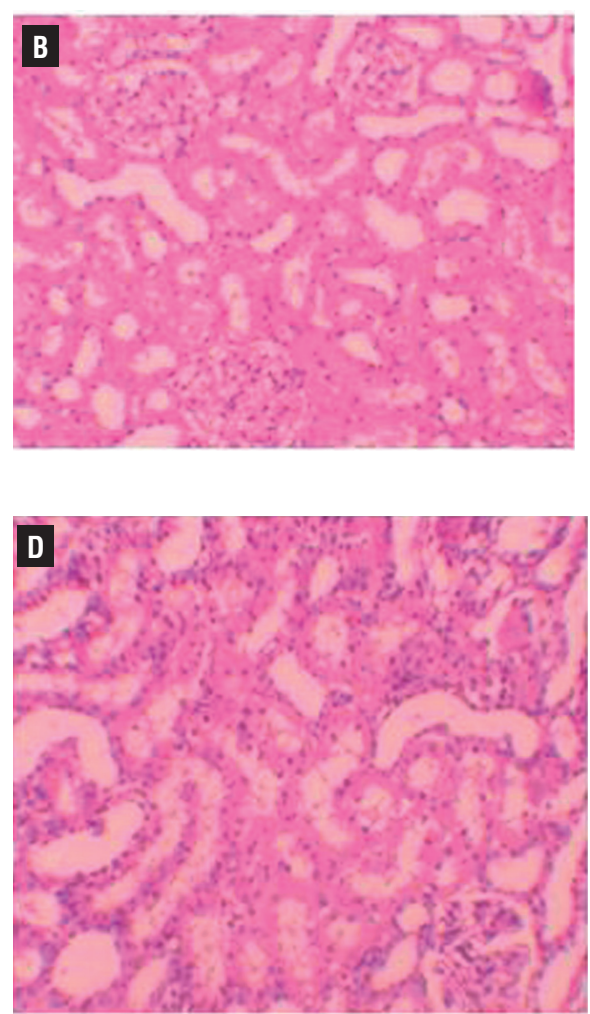
Figure 2 - A) severe mononuklear leukocyte infiltration in the cortex of UUO group (hematoxylin \& eosin $x 400$ ) B) leukocyte infiltration was observed in the peritubular interstitium of the UUO (hematoxylin \& eosin x400); c) leukocyte infiltration was reduced in the NaHS-treated group (hematoxylin \& eosin x400).
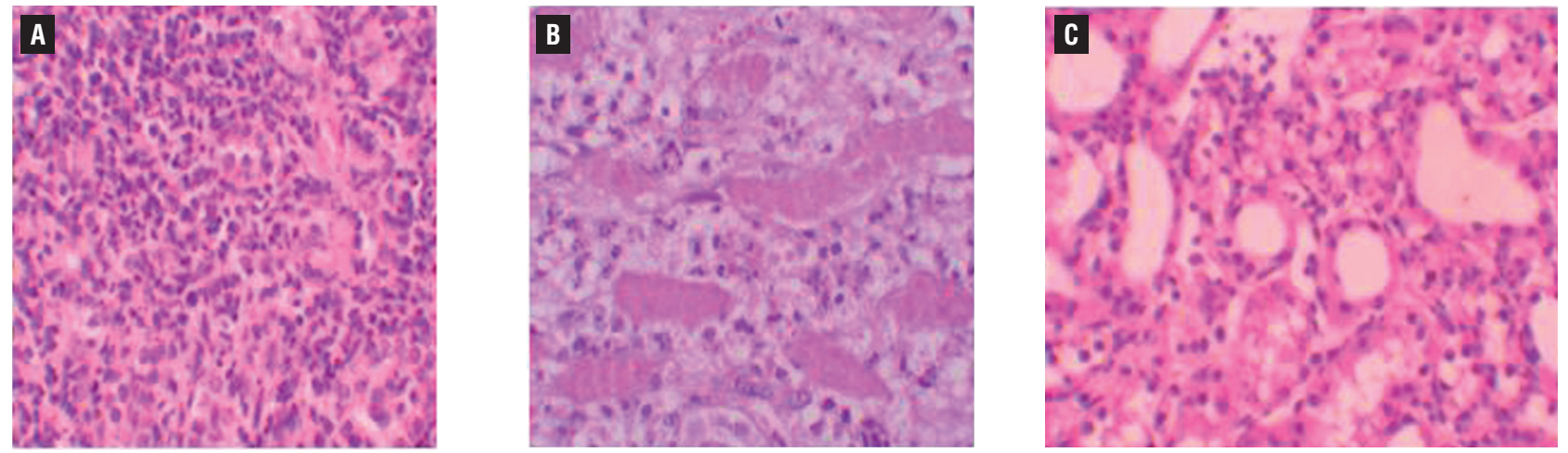

the glomerular filtration rate drop. Within a few days, hydronephrosis starts to develop, followed by interstitial inflammatory infiltration, apoptosis, and necrosis.

The pathogenesis of renal fibrosis caused by UUO involves infiltration of the kidney by inflammatory cells including monocytes, activation and possible transformation of intrinsic renal cells, and interactions between infiltrating and resident cells. Reactive oxygen species (ROS) are a recently recognized mechanism in the pathogenesis of UUO in experimental studies (17). So we decided to measure the MDA, GSH, and nitric oxide (NO) content, as a means of oxidative stress. In our study confirmed through a quantitative survey the protective role of $\mathrm{H}_{2} \mathrm{~S}$ on renal tissue damage after the induction of UUO in rats. Our results showed that the obstructed kidney had significantly higher tissue MDA, NO levels, and lower GSH levels along with more fibrosis. Our findings corroborate those of earlier studies demonstrating that an enhanced endogenous oxidative stress has a major role in the severity of UUO-induced acute renal failure $(18,19)$. On the other hand, $\mathrm{H}_{2} \mathrm{~S}$ reduced the severity of injury, depressed the concentration of these cytokines and increased the antioxidative capacity.

Endogenous $\mathrm{H}_{2} \mathrm{~S}$ has been proposed as a novel cytoprotective mediator (20), and there is growing evidence of direct and indirect antioxidant effects of $\mathrm{H}_{2} \mathrm{~S}$. In cell culture experiments, $\mathrm{H}_{2} \mathrm{~S}_{\text {HS }}^{-}$generated from NaHS has been shown to

Figure 3 - A) normal kidney morphology in a sham group, group (masson \& trichrome x200). b) severe fibrosis was observed in the peritubular interstitium of the UUO, group (masson \& trichrome x400). c) mild fibrosis was reduced in the NaHS-treated group (masson \& trichrome $\mathbf{4 0 0}$ ).
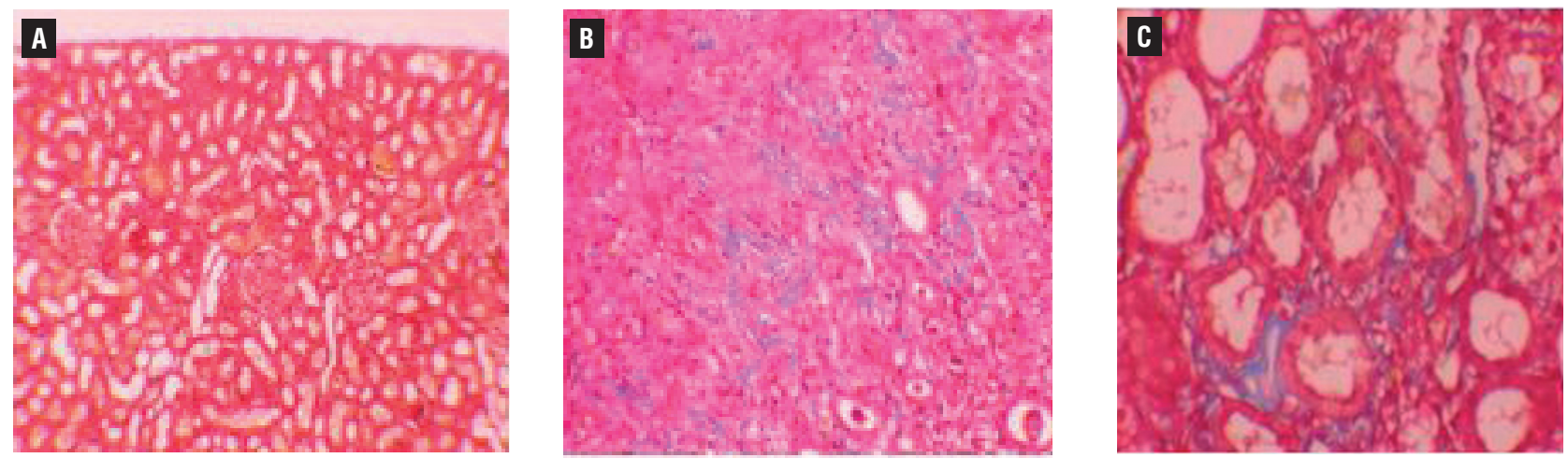
Table 3 - Semiquantitative analysis of tubular necrosis, interstitial fibrosis, mononuclear cell infiltration in control, Sham, $\mathrm{UO}$, and $\mathrm{UO}+\mathrm{H}_{2} \mathrm{~S}$ treated rats.

\begin{tabular}{|c|c|c|c|c|c|c|c|c|c|c|c|c|c|}
\hline & \multicolumn{4}{|c|}{ Tubular necrosis } & \multicolumn{5}{|c|}{ Interstitial fibrosis } & \multicolumn{4}{|c|}{ Mononuclear cell infiltration } \\
\hline & $\mathrm{n}$ & 0 & 1 & 2 & 3 & 0 & 1 & 2 & 3 & 0 & 1 & 2 & 3 \\
\hline Control & 6 & 6 & 0 & 0 & 0 & 6 & 0 & 0 & 0 & 5 & 1 & 0 & 0 \\
\hline Sham & 6 & 6 & 0 & 0 & 0 & 6 & 0 & 0 & 0 & 5 & 1 & 0 & 0 \\
\hline UO & 6 & 0 & 0 & 4 & 2 & 0 & 1 & 3 & 2 & 0 & 1 & 3 & 2 \\
\hline $\mathrm{UO}+\mathrm{NaHS}^{\mathrm{b}}$ & 6 & 1 & 3 & 2 & 0 & 2 & 3 & 1 & 0 & 1 & 1 & 4 & 0 \\
\hline
\end{tabular}

Score 0: no degeneration, 1: mild degeneration, 2: moderate degeneration, and 3: severe degeneration

a Statistical significant difference from the Sham group

${ }^{b}$ Statistical significant difference from the U0 group and $\mathrm{P}<0.05$.

'scavenge' detrimental pro-inflammatory oxidants, such as $\mathrm{H}_{2} \mathrm{O}_{2}$ (21), $\mathrm{ClO}^{-}$(22), superoxide, $\mathrm{ONOO}^{-}$and NO, inhibit cell death induced by these mediators as well as prevent oxidative modification of intracellular proteins (22) and LDL (low-density lipoprotein) (23). In neuronal cells, NaHS inhibited cell death induced by $\beta$-amyloid, mediated at least in part via antioxidant effects (24) and up-regulating intracellular glutathione synthesis through increasing cysteine uptake and elevating $\gamma$-glutamylcysteine synthetase activity. NaHS is also reported to degrade lipid peroxides (24), inhibit the expression and activity of NADPH oxidase and up-regulate thioredoxin-1 expression in vascular endothelial cells (25). Increased hepatic GSH synthesis and decreased lipid peroxidation are also observed with $\mathrm{NaHS}$ treatment in a murine hepatic ischaemia/reperfusion injury model (26).

Increased lipid peroxidation (LPO) has been reported in renal cortexes by the induction of excessive ROS in renal ischemic reperfusion (27). MDA is the product in the LPO process and is widely used as a reliable marker of tissue damage. In the present study, we found increased MDA levels in UUO group and as protective effect of $\mathrm{H}_{2} \mathrm{~S}$ lower MDA levels in group determined by UUO+NaHS. The GSH antioxidant system is considered the most notable cellular protective mechanism. GSH has a very important role in protecting against oxygen free radical damage by providing reducing equivalents for several enzymes, as well as scavenging hydroxyl radicals and singlet oxygen. Its depletion is a common consequence of increased formation of ROS like UUO- induced nephrotoxicity. In group given UUO+NaHS, we found increased GSH levels. However, our study have shown that $\mathrm{H}_{2} \mathrm{~S}$ effects NO levels protectively similar to some previous studies with different antioxidant agents (28). $\mathrm{H}_{2} \mathrm{~S}$ can inhibit NO production and NF-kappaB activation in LPS-stimulated macrophages through a mechanism that involves the action of HO-1/CO (29). Because of that, in our study we found decreased NO levels in UUO+NaHS group compared to UUO group. These findings strongly indicate that $\mathrm{H}_{2} \mathrm{~S}$ is important in protecting the kidney from UUO-induced injury through improvement in oxidant status.

In this study, the histopathologic examination of kidneys showed severe and extensive damage in UUO rats which have tubular necrosis and edema. This could be due to the formation of highly reactive radicals as a consequence of oxidative stress caused by UUO. The kidneys of the control group showed normal histological features, but the UUO group revealed more extensive and marked tubular necrosis. On the other hand, the tubules from rats of the UUO+NaHS group were nearly normal in histological appearance except for a slight desquamation and atrophy of the tubular epithelial cells. Similar changes were also reported by some studies who demonstrated structural changes in renal tissue of gentamicin-treated animals and its reversal by various agents (30). 
In conclusion, the results reported here indicate that $\mathrm{H}_{2} \mathrm{~S}$ exerts a preventive effect on UUO-induced kidney damage in rats by reducing oxidative stress. At present, hydronephrosis is an urological condition that needs a quick surgical treatment. But, surgery pre-operative phase might take longer than we planned. So, we can use NaHS treatment to prevent the kidney damage in clinics until the surgical treatment. One limitation in our study was that the underlying molecular mechanisms that are responsible for the positive effects of NaHS are yet to be determined. We therefore propose that NaHS supplementation therapy can be used for kidney protection in patients with UUO, such as with ureteral stones. Hovewer, further animal and clinical studies are needed to confirm our suggestion.

\section{CONFLICT OF INTEREST}

None declared.

\section{REFERENCES}

1. Yeh $\mathrm{CH}$, Chiang HS, Lai TY, Chien CT. Unilateral ureteral obstruction evokes renal tubular apoptosis via the enhanced oxidative stress and endoplasmic reticulum stress in the rat. Neurourol Urodyn. 2011;30:472-9.

2. Dendooven A, Ishola DA Jr, Nguyen TQ, Van der Giezen DM, Kok RJ, Goldschmeding R, et al. Oxidative stress in obstructive nephropathy. Int J Exp Pathol. 2011;92:202-10.

3. Uyeturk U, Terzi EH, Kemahli E, Gucuk A, Tosun M, Çetinkaya A. Alleviation of kidney damage induced by unilateral ureter obstruction in rats by Rhodiola rosea. $\mathrm{J}$ Endourol. 2013;27:1272-6.

4. Zecher M, Guichard C, Velásquez MJ, Figueroa G, Rodrigo R. Implications of oxidative stress in the pathophysiology of obstructive uropathy. Urol Res. 2009;37:19-26.

5. Song K, Wang F, Li Q, Shi YB, Zheng HF, Peng $H$, et al. Hydrogen sulfide inhibits the renal fibrosis of obstructive nephropathy. Kidney Int. 2014;85:1318-29.

6. Chevalier RL, Thornhill BA, Chang AY. Unilateral ureteral obstruction inneonatal rats leads to renal insufficiency in adulthood. Kidney Int. 2000;58:1987-95.

7. Acikgoz Y, Can B, Bek K, Acikgoz A, Ozkaya O, Genç G, et al. The effect of simvastatin and erythropoietin on renal fibrosis in rats with unilateral ureteral obstruction. Ren Fail. 2014:36:252-7.
8. Kawada N, Moriyama T, Ando A, Fukunaga M, Miyata T, Kurokawa $\mathrm{K}$, et al. Increased oxidative stress in mouse kidneys with unilateral ureteral obstruction. Kidney Int. 1999;56:1004-13.

9. Klahr S. Urinary tract obstruction. Semin Nephrol. 2001;21:133-45.

10. Jung KJ, Jang HS, Kim JI, Han SJ, Park JW, Park KM. Involvement of hydrogen sulfide and homocysteine transsulfuration pathway in the progression of kidney fibrosis after ureteral obstruction. Biochim Biophys Acta. 2013;1832:1989-97.

11. Kimura H. Hydrogen sulfide: its production, release and functions. Amino Acids. 2011;41:113-21.

12. Szabó C. Hydrogen sulphide and its therapeutic potential. Nat Rev Drug Discov. 2007;6:917-35.

13. Bracht H, Scheuerle A, Gröger M, Hauser B, Matallo J, McCook 0 , et al. Effects of intravenous sulfide during resuscitated porcine hemorrhagic shock*. Crit Care Med. 2012 Jul;40(7):2157-67. Erratum in: Crit Care Med. 2013;41:e41. Dosage error in article text.

14. Yan H, Du J, Tang C. The possible role of hydrogen sulfide on the pathogenesis of spontaneous hypertension in rats. Biochem Biophys Res Commun. 2004;313:22-7.

15. Ozbek E, Ilbey YO, Ozbek M, Simsek A, Cekmen M, Somay A. Melatonin attenuates unilateral ureteral obstruction-induced renal injury by reducing oxidative stress, iNOS, MAPK, and NF-kB expression. J Endourol. 2009;23:1165-73.

16. Allen CT . Laboratory methods in histochemistry. In: Prophet EB, Mills B, Arrington JB, Sobin LH (eds) American registry of pathology, 1st edn. Washington DC, 1992; pp. 53.

17. Kinugasa F, Noto T, Matsuoka H, Urano Y, Sudo Y, Takakura $S$, et al. Prevention of renal interstitial fibrosis via histone deacetylase inhibition in rats with unilateral ureteral obstruction. Transpl Immunol. 2010;23:18-23.

18. Jiang D, Zhang Y, Yang M, Wang S, Jiang Z, Li Z. Exogenous hydrogen sulfide prevents kidney damage following unilateral ureteral obstruction. Neurourol Urodyn. 2014;33:538-43.

19. Martínez-Salgado C, López-Hernández FJ, López-Novoa JM. Glomerular nephrotoxicity of aminoglycosides. Toxicol Appl Pharmacol. 2007;223:86-98.

20. Walker PD, Shah SV. Gentamicin enhanced production of hydrogen peroxide by renal cortical mitochondria. Am J Physiol. 1987;253(4 Pt 1):C495-9.

21. Whiteman M, Moore PK. Hydrogen sulfide and the vasculature: a novel vasculoprotective entity and regulator of nitric oxide bioavailability? J Cell Mol Med. 2009;13:488-507.

22. Muzaffar S, Shukla N, Bond M, Newby AC, Angelini GD, Sparatore A, et al. Exogenous hydrogen sulfide inhibits superoxide formation, NOX-1 expression and Rac1 activity in human vascular smooth muscle cells. J Vasc Res. 2008;45:521-8. 
23. Whiteman M, Cheung NS, Zhu YZ, Chu SH, Siau JL, Wong BS, et al. Hydrogen sulphide: a novel inhibitor of hypochlorous acid-mediated oxidative damage in the brain? Biochem Biophys Res Commun. 2005;326:794-8.

24. Muellner MK, Schreier SM, Laggner H, Hermann M, Esterbauer $\mathrm{H}$, Exner $\mathrm{M}$, et al. Hydrogen sulfide destroys lipid hydroperoxides in oxidized LDL. Biochem J. 2009;420:277-81.

25. Liu YY, Bian JS. Hydrogen sulfide protects amyloid- $\beta$ induced cell toxicity in microglia. J Alzheimers Dis. 2010;22:1189200. Erratum in: J Alzheimers Dis. 2012;31:453.

26. Vacek TP, Gillespie W, Tyagi N, Vacek JC, Tyagi SC. Hydrogen sulfide protects against vascular remodeling from endothelial damage. Amino Acids. 2010;39:1161-9.

27. Jha S, Calvert JW, Duranski MR, Ramachandran A, Lefer DJ. Hydrogen sulfide attenuates hepatic ischemia-reperfusion injury: role of antioxidant and antiapoptotic signaling. Am J Physiol Heart Circ Physiol. 2008;295:H801-6.

28. Ozbek E, Cekmen M, Ilbey YO, Simsek A, Polat EC, Somay A. Atorvastatin prevents gentamicin-induced renal damage in rats through the inhibition of p38-MAPK and NF-kappaB pathways. Ren Fail. 2009;31:382-92.
29. Oh GS, Pae HO, Lee BS, Kim BN, Kim JM, Kim HR, et al. Hydrogen sulfide inhibits nitric oxide production and nuclear factor-kappaB via heme oxygenase-1 expression in RAW264.7 macrophages stimulated with lipopolysaccharide. Free Radic Biol Med. 2006;41:106-19.

30. Kumar KV, Shifow AA, Naidu MU, Ratnakar KS. Carvedilol: a beta blocker with antioxidant property protects against gentamicin-induced nephrotoxicity in rats. Life Sci. 2000;66:2603-11.
Correspondence address: Murat Dursun, MD Bahcelievler State Hospital Department of Urology Kocasinan Cavusbası Str. 34192, Bahcelievler, Istanbul, Turkey Fax: + 90212 496-7000 E-mail:mrt_drsn@hotmail.com 